\title{
Risk of Osteonecrosis of the Jaw in Patients with Postmenopausal Osteoporosis: A Systematic Literature Review
}

\author{
Gloria Candelas ${ }^{1 *}$, Virginia Villaverde ${ }^{2}$, Petra Dïaz ${ }^{3}$, Mercedes Guerra $^{3}$ and Antonio Naranjo 4 \\ ${ }^{1}$ Rheumatology Unit, Hospital Clínico San Carlos, Madrid, Spain \\ ${ }^{2}$ Rheumatology Unit, Hospital Universitario de Móstoles, Spain \\ ${ }^{3}$ Investigation Unit, Spanish Society of Rheumatology, Spain \\ ${ }^{4}$ Rheumatology Unit, Hospital Universitario de Gran Canaria Doctor Negrín, Las Palmas de Gran Canaria, Spain \\ *Corresponding author: Gloria Candelas, Rheumatology Unit, Hospital Universitario Clínico de San Carlos, Calle Profesor \\ Martín Lagos s/n 28040, Madrid, Spain, Tel: +34-916303615
}

\begin{abstract}
Objective: To systematically review the literature available on the use of Denosumab (Dmab) in osteoporosis and the risk of ONJ.

Methods: The studies retrieved by the above strategies were included if they met the following pre-established criteria. The patients studied had to be diagnosed of osteoporosis (all ages, both sexes), and they had to be taking Dmab. We measured the likelihood of having osteonecrosis of the jaw. We included in the search for systematic reviews randomised clinical trials (RCT) and cohort studies. We excluded articles on cancer or other disease other than osteoporosis, animal and basic science studies. There was no limitation with regard to the number of patients included in the studies. The bibliographic databases screened were Medline and Embase and the Cochrane. The searches were conducted with language restriction (English, French and Spanish).
\end{abstract}

Results: We found 10 articles that were studied in detail because by title or abstract they seemed to be related to the study, or because they had no abstract to review. Finally, 2 studies were included (one of them by indirect search) (Table 1). One of the study included was case series and another was systematic review of cases and series of cases and their data retrieved. The quality level of the two articles was low. Related to the studies population, all patients had osteoporosis, mostly were women with mean ages around 50-78 years. All patients were treated with subcutaneous denosumab every 6 months.

In these two studies, the incidence of ONJ was very low. Some systemic factors such as previous treatment with BPs or dental extractions are associated with the development of osteonecrosis of the jaws in patients treated with
Dmab. Stage 1 of ONJ was the most common and conservative treatment was used with the healing of the lesions.

Conclusions: There are not enough studies that have evaluated the role of Dmab in the development of osteonecrosis (ONJ) of the jaws. Some systemic factors that have been associated with the development of ONJ are described in patients treated with Dmab, such as previous treatment with bisphosphonates or tooth extractions.

\section{Introduction}

Osteoporosis is a disease characterized by decreased bone mass, microarchitectural deterioration of the skeleton and impaired bone strength which results from increased bone resorption relative to formation. Anti-resorptive drugs significantly reduce bone turnover, providing an increase in $\mathrm{BMD}$ and a reduction in risk of fracture [1].

Recent advances have identified the Receptor Activator for Nuclear Factor -B Ligand (RANKL) as a critical mediator of bone remodeling. RANKL is essential for the formation, function, and survival of the osteoclasts. It binds to its cognate receptor RANK on the surface of precursors and mature osteoclasts, and stimulates these cells to mature and resorb bone.

Dmab is a novel antiresorptive agent that also inhibits osteclast-mediated bone resorption but works through a different pathway than bisphosphonates.

Citation: Candelas G, Villaverde V, Dïaz P, Guerra M, Naranjo A (2019) Risk of Osteonecrosis of the Jaw in Patients with Postmenopausal Osteoporosis: A Systematic Literature Review. Clin Arch Bone Joint Dis 2:010. doi.org/10.23937/2643-4091/1710010

Accepted: July 23, 2019: Published: July 25, 2019

Copyright: (C) 2019 Candelas G, et al. This is an open-access article distributed under the terms of the Creative Commons Attribution License, which permits unrestricted use, distribution, and reproduction in any medium, provided the original author and source are credited. 
Table 1: Main characteristics of the included studies.

\begin{tabular}{|c|c|c|c|c|c|}
\hline Reference & Study & Population & $\begin{array}{l}\text { Follow-Up } \\
\text { Intervention }\end{array}$ & Results & $\begin{array}{l}\text { Quality Of } \\
\text { Evidence } \\
\text { (Sign) }\end{array}$ \\
\hline Bagan [7] & $\begin{array}{l}\text { Objectives: } \\
\text { Describe clinical } \\
\text { characteristics } \\
\text { and the evolution } \\
\text { of a series of } \\
\text { patients who } \\
\text { develop ONJ } \\
\text { after treatment } \\
\text { for osteoporosis } \\
\text { (OP) with } \\
\text { denosumab } \\
\text { (Dmab) } \\
\text { Search period: } \\
\text { not specified }\end{array}$ & $\begin{array}{l}\text { N: } 10 \text { patients } \\
\text { Mean age: } 73.7 \pm 11.6 \\
\text { years } \\
\text { Sex: } 100 \% \text { women } \\
\text { Exclusion criteria } \\
\text { were treatment with } \\
\text { other medications } \\
\text { or chemotherapy for } \\
\text { associated cancers }\end{array}$ & $\begin{array}{l}\text { Intervention: } \\
\text { Treatment } \\
\text { with Dmab (60 } \\
\text { mg)/6 months } \\
\text { Follow-up } \\
\text { period: } \\
\text { not specified }\end{array}$ & $\begin{array}{l}\text {-The average number of doses of } \\
\text { denosumab was } 3.4 \pm 2.2 \text {. } \\
9 \text { patients ( } 90 \% \text { ) had also been } \\
\text { treated before with oral BPs, for an } \\
\text { average of } 44.7 \pm 25.11 \text { months ( } 4 \\
\text { with ibandronate, } 3 \text { with risedronate } \\
\text { and } 3 \text { with alendronate. } \\
\text {-In } 7 \text { ( } 70 \%) \text {, the ONJ was located in } \\
\text { the jaw. } \\
\text { - The most common local risk } \\
\text { factor found was tooth extraction } \\
\text { (six cases, } 60 \%) \text {; Only one case } \\
\text { associated with dental implant was } \\
\text { found. Three cases ( } 30 \%) \text { had an } \\
\text { intraoral and necrotic fistula and in } \\
9 \text { cases ( } 90 \% \text { ) bone exposure was } \\
\text { observed. } \\
\text { - Bone sclerosis was present in all } \\
\text { cases. No sequestration was found } \\
\text { in any case, osteolysis was present } \\
\text { in six ( } 60 \% \text { ) cases. } \\
\text { - Stage } 1 \text { of ONJ was the most } \\
\text { common, occurring in eight ( } 80 \% \text { ) } \\
\text { cases, and in all cases except one, } \\
\text { conservative treatment was used } \\
\text { with the healing of the lesions }\end{array}$ & 3 \\
\hline Oliveira [6] & $\begin{array}{l}\text { Design: } \\
\text { Systematic } \\
\text { review of } \\
\text { cases and } \\
\text { series of cases } \\
\text { Objectives: } \\
\text { Review the } \\
\text { literature of } \\
\text { clinical cases } \\
\text { that develop } \\
\text { ONM after } \\
\text { treatment with } \\
\text { Dmab } \\
\text { Search period: } \\
\text { January } 2010 \text { to } \\
\text { May } 2015\end{array}$ & $\begin{array}{l}\text { N:17 patients } \\
8 \text { OP (mean age: } \geq 60 \\
\text { (58-78a). } 100 \% \text { women. } \\
9 \text { Cancer. } \\
\text { To be included, the } \\
\text { articles had to be a case } \\
\text { or a series of clinical } \\
\text { cases in patients } \geq 18 \\
\text { years of who used Dmab } \\
\text { for the treatment of } \\
\text { osteoporosis including } \\
\text { patients with Cancer and } \\
\text { who had received some } \\
\text { therapy for ONJ. }\end{array}$ & $\begin{array}{l}\text { Treatment } \\
\text { with Dmab (60 } \\
\text { mg)/6 months }\end{array}$ & $\begin{array}{l}\text {-Of the } 17 \text { cases detected only } 8 \\
\text { were in patients with osteoporosis } \\
\text { and osteopenia. } \\
\text {-The majority of cases of ONJ } \\
\text { occurred in the posterior mandibular } \\
\text { area and only one of them in the } \\
\text { posterior maxillary area. } \\
\text {-The associated systemic factors } \\
\text { were: } \\
\text {-Previous appointment with BPs (in } \\
6 \text { of } 8 \text { cases). } \\
\text {-Dental extractions ( } 4 \text { of the } 8 \\
\text { cases). }\end{array}$ & 3 \\
\hline
\end{tabular}

Dmab is a fully human monoclonal antibody (IgG2) that inhibits RANKL with high specificity, mimicking the effects of OPG on RANKL.6 [2].

Following menopause, increased RANKL results in increased bone resorption and bone loss, which can lead to osteoporosis, a condition characterized by compromised bone strength and increased risk of fracture [3]. In postmenopausal women with osteoporosis, denosumab significantly reduced bone turnover markers (BTMs), increased bone mineral density (BMD), and reduced new vertebral (68\%), hip (40\%), and nonvertebral (20\%) fractures compared with placebo [4].

The American Association of Oral and Maxillofacial Surgeons (AAOMS) has recently (2014) updated their definition of medication-related ONJ to (1) Current or previous treatment with antiresorptive or antiangiogenic agents; (2) Exposed bone or bone that can be probed through an intraoral or extraoral fistula (e) in the maxillofacial area that has persisted for more than 8 weeks; and (3) No history of radiation therapy to the jaws or obvious metastatic disease to the jaws [5].

The ONJ is a multifactorial disease, of which occurrence is predisposed by some factors. It includes local factors such as tooth extraction, dent alveolar surgery, periodontal disease, trauma from ill-fitting dentures [6] and systemical factors such as malignant diseases (breast, lung and prostate, multiple myeloma), chemotherapy, chronic steroid therapy, smoking, diabetes and anaemia [6]. 
Table 2: Search strategies in the different bibliographic databases and hits.

\section{PUBMED}

Search $(((((($ "osteoporosis"[MeSH Terms] OR "osteoporoses"[Title/Abstract] OR "post traumatic osteoporosis"[Title/Abstract] OR post traumatic osteoporoses[Title/Abstract] OR "osteoporosis senile"[Title/Abstract] OR "senile osteoporosis"[Title/Abstract] OR "bone loss age related"[Title/Abstract] OR "age related bone loss"[Title/Abstract] OR "age related bone losses"[Title/Abstract] OR "bone loss, osteoporosis"[Title/Abstract] OR "age related osteoporosis"[Title/Abstract] OR "age related osteoporoses"[Title/ Abstract] OR "osteoporosis, postmenopausal"[MeSH Terms] OR "perimenopausal bone loss"[Title/Abstract] OR "bone loss postmenopausal"[Title/Abstract] OR "post menopausal osteoporosis"[Title/Abstract] OR "postmenopausal osteoporosis"[Title/ Abstract] OR "postmenopausal osteoporoses"[Title/Abstract] OR "postmenopausal bone loss"[Title/Abstract] OR "female athlete triad syndrome"[MeSH Terms] OR "female athlete triad"[Title/Abstract] OR "decalcification, pathologic"[MeSH Terms] OR "pathologic decalcification"[Title/Abstract] OR "corticosteroid induced osteoporosis"[Title/Abstract] OR "glucocorticoid induced osteoporosis"[Title/Abstract] OR "idiopathic osteoporosis"[Title/Abstract] OR "involutional osteoporosis"[Title/Abstract] OR "juvenile osteoporosis"[Title/Abstract] OR "primary osteoporosis"[Title/Abstract] OR "secondary osteoporosis"[Title/Abstract] OR "bone density"[MeSH Terms] OR "bone densities"[Title/Abstract] OR "density, bone"[Title/Abstract] OR "bone mineral density"[Title/Abstract] OR "bone mineral densities"[Title/Abstract] OR "density, bone mineral"[Title/Abstract] OR "bone mineral content"[Title/Abstract] OR "bone mineral contents"[Title/Abstract] OR "bmd"[Title/Abstract] OR "fractures, bone"[MeSH Terms] OR "broken bones"[Title/Abstract] OR "broken bone"[Title/Abstract] OR "bone fractures"[Title/Abstract] OR "bone fracture"[Title/ Abstract] OR "fracture, bone"[Title/Abstract] OR "bone mineral density"[Title/Abstract] OR "low bone mass"[Title/Abstract] OR "low bone mass density"[Title/Abstract] OR "low bone mineral density"[Title/Abstract] OR "low bone"[Title/Abstract] OR "low bone density"[Title/Abstract] OR "postmenopausal bone loss"[Title/Abstract] OR "bone loss osteoporosis"[Title/Abstract] OR "bone loss postmenopausal"[Title/Abstract] OR "bone loss"[Title/Abstract])) NOT cancer [sb])) AND (("Denosumab"[Mesh] OR "denosumab"[Title/Abstract] OR "prolia"[Title/Abstract] OR "xgeva"[Title/Abstract] OR "amg 162"[Title/Abstract] OR "amg162"[Title/Abstract]))) AND ((((((("Jaw Diseases/chemically induced"[Mesh] OR "Jaw Diseases/drug effects"[Mesh] OR "Jaw Diseases/growth and development"[Mesh] OR "Jaw Diseases/pathology"[Mesh] OR "Jaw Diseases/pharmacology"[Mesh]))) OR (("Maxillary Diseases/chemically induced"[Mesh] OR "Maxillary Diseases/chemistry"[Mesh] OR "Maxillary Diseases/drug therapy"[Mesh] OR "Maxillary Diseases/pathology"[Mesh]))) OR (("osteomyelitis/pathology"[MeSH Terms] OR "osteomyelitis/ chemically induced"[MeSH Terms] OR "osteomyelitis/secondary"[MeSH Terms] OR "osteomyelitis/drug therapy"[MeSH Terms]))) OR (("periodontal attachment loss/chemically induced"[MeSH Terms] OR "periodontal attachment loss/drug therapy"[MeSH Terms] OR "periodontal attachment loss/pathology"[MeSH Terms]))) OR (("Periodontal Diseases/chemically induced"[Mesh] OR "Periodontal Diseases/chemistry"[Mesh] OR "Periodontal Diseases/drug effects"[Mesh] OR "Periodontal Diseases/injuries"[Mesh] OR "Periodontal Diseases/pathology"[Mesh] OR "Periodontal Diseases/secondary"[Mesh]))) OR (("Periodontitis/chemically induced"[Mesh] OR "Periodontitis/drug effects"[Mesh] OR "Periodontitis/pathology"[Mesh]))))) NOT (("Animals"[Mesh] NOT ("Animals"[Mesh] AND "Humans"[Mesh])))

\section{EMBASE PICO2b}

'osteoporosis'/exp OR 'decalcification, pathologic' OR 'endocrine osteoporosis' OR 'osteoporosis' OR 'osteoporotic decalcification' AND ('denosumab'/exp OR 'amg 162' OR 'amg162' OR 'amgiva' OR 'denosumab' OR 'prolia' OR 'xgeva') AND ('jaw disease'/exp OR 'jaw disease' OR 'jaw diseases' OR 'jaw disorder' OR 'mandible injury' OR 'mandibular diseases' OR 'mandibular injuries' OR 'mandibular injury' OR 'maxillary diseases' OR 'temporomandibular disorder' OR 'osteomyelitis'/exp OR 'osteomyelitis' OR 'periodontal disease'/exp OR 'dental loss' OR 'dental migration' OR 'dental mobility' OR 'edentulism' OR 'furcation defects' OR 'mesial movement of teeth' OR 'paradontal disease' OR 'paradontopathy' OR 'paraodontopathy' OR 'parodentopathy' OR 'parodontal disease' OR 'parodontium disease' OR 'parodontive tissue disease' OR 'peridontal disease' OR 'peridontal tissue disease' OR 'peridontium disease' OR 'periodontal atrophy' OR 'periodontal attachment loss' OR 'periodontal cyst' OR 'periodontal disease' OR 'periodontal diseases' OR 'periodontal infection' OR 'periodontium disease' OR 'periodontopathy' OR 'tooth loss' OR 'tooth migration' OR 'tooth mobility' OR 'tooth movement' OR 'periodontitis'/exp OR 'paradontitis' OR 'parodontitis' OR 'peridontitis' OR 'periodontitis') AND ('clinical trial'/de OR 'controlled clinical trial'/de OR 'controlled study'/de OR 'drug dose comparison'/de OR 'human'/de OR 'phase 3 clinical trial'/de OR 'phase 3 clinical trial (topic)'/de OR 'practice guideline'/de OR 'randomized controlled trial'/de OR 'randomized controlled trial (topic)'/de OR 'systematic review'/de) AND 'review'/it AND ([english]/lim OR [french]/lim OR [spanish]/lim)

PICO 2b Cochrane:

\begin{tabular}{|l|l|l|}
\hline$\# 1$ & MeSH descriptor: [Osteoporosis] explode all trees & 3542 \\
\hline$\# 2$ & MeSH descriptor: [Neoplasms] explode all trees & 61198 \\
\hline$\# 3$ & $\# 1$ not \#2 & 3360 \\
\hline$\# 4$ & MeSH descriptor: [Denosumab] explode all trees & 115 \\
\hline$\# 5$ & MeSH descriptor: [Jaw Diseases] explode all trees and with qualifier(s): [Chemically induced - Cl] & 19 \\
\hline$\# 6$ & MeSH descriptor: [Maxillary Diseases] explode all trees & 0 \\
\hline$\#$ \#8 & MeSH descriptor: [Osteomyelitis] explode all trees & 134 \\
\hline$\# 9$ & MeSH descriptor: [Periodontal Diseases] explode all trees & 734 \\
\hline$\# 10$ & MeSH descriptor: [Periodontitis] explode all trees & 4510 \\
\hline$\# 11$ & $\# 5$ or \#6 or \#7 or \#8 or \#9 or \#10 & 2100 \\
\hline \#12 & $\# 3$ and \#4 and \#11 & 4682 \\
\hline
\end{tabular}


The aim of this study was, therefore, to systematically review the literature available on the use of Dmab in osteoporosis and the risk of ONJ. This information was afterwards examined and used by the experts of the Spanish Society of Rheumatology Consensus of osteoporosis to generate clinical practice recommendations for rheumatologists.

\section{Methods}

As a part of the Spanish Society of Rheumatology Consensus of osteoporosis, a systematic literature review was performed to address the experts' question on the risk of osteonecrosis of the jaw in patients with postmenopausal osteoporosis. A protocol of the review was established and further advice from the complete team of the Consensus was obtained.

\section{Search strategy}

The studies were identified by sensitive search strategies in the main bibliographic databases (Table 2). For this purpose, an expert librarian collaborated and checked the search strategies.

The following bibliographic databases were screened as follows: Medline and Embase from the beginning to 8th May 2017, and the Cochrane Central reg- ister of Controlled Trials (CENTRAL) up to $8^{\text {th }}$ May 2017. The searches were conducted with a language restriction (English, French and Spanish).

In the end, a hand search was completed by reviewing the references of the included studies, and all the publications or other information provided by the experts related to the systematic review were also examined.

\section{Selection criteria}

The studies retrieved by the above strategies were included if they met the following pre-established criteria. The patients studied had to be diagnosed of osteoporosis (all ages, both sexes), and they had to be taking Dmab. We measured the likelihood of having osteonecrosis of the jaw.

We included in the search for systematic reviews randomised clinical trials (RCT) and cohort studies. We excluded articles on cancer or other disease other than osteoporosis, animal and basic science studies. There was no limitation with regard to the number of patients included in the studies. Selection of studies and data collection EndNote X7s software was used to manage the records retrieved by searches of the different electronic data bases and manual search methods.

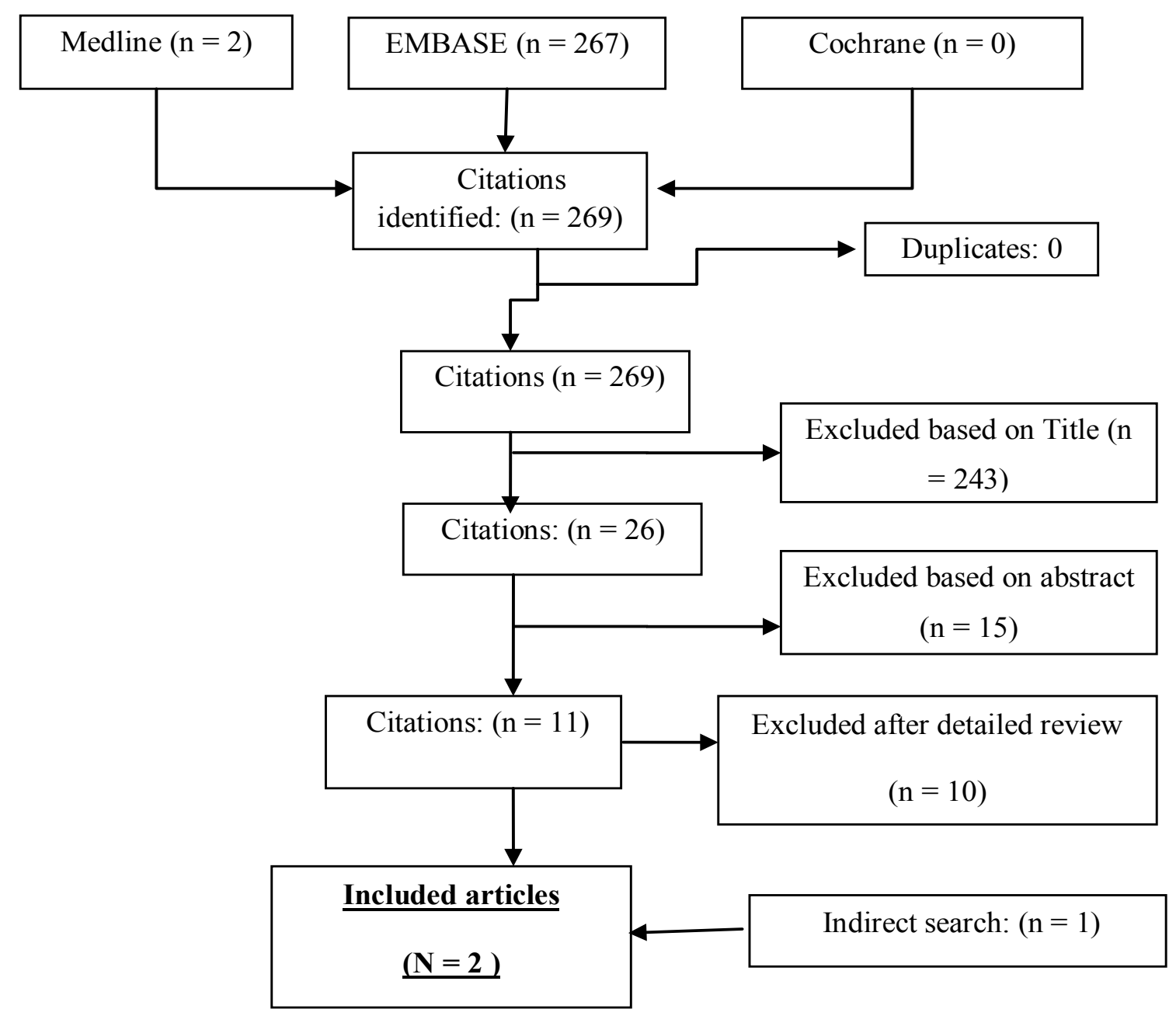

Figure 1: Articles retrieved by the different search strategies and result of selection and appraisal process. 


\section{Screening of studies, data collection and analysis}

One reviewer (G.C) screened the titles and abstracts of the retrieved articles for selection criteria independently. The reviewer collected the data from the studies included by using ad hoc standard forms. All collection was double by article and independent. The reviewer entered the data from the forms into spreadsheets. Articles that did not fulfil all the inclusion criteria or that had insufficient data were excluded. To grade the quality, we used the levels of scientific evidence and SIGN recommendation grades. Metaanalysis was only planned in case enough homogeneity was present among the included studies.

\section{Results}

The result of the search strategies is presented in Table 2 by specific terms, and in total in Figure 1. We found 10 articles that were studied in detail because by title or abstract they seemed to be related to the study, or because they had no abstract to review. Table 3 shows the studies that were excluded after detailed review and the reasons for exclusion. Finally, 2 studies were included (one of them by indirect search) (Table 1 ). One of the study included was case series and another was systematic review of cases and series of cases and their data retrieved. The quality level of the two articles was 3 . Table 1 shows the main characteristics of the included studies.

Related to the studies population, all patients had osteoporosis, mostly were women with mean ages around $50-78$ years. All patients were treated with subcutaneous Dmab every 6 months.

In the study of Bagan, et al. [7] all patients with osteoporosis treated with Dmab at a dose of $60 \mathrm{mg}$ subcutaneously every 6 months were included. Exclusion criteria were treatment with other medications or chemotherapy for associated cancers. In each case, in addition to age and sex, Dmab doses were recorded, and in patients who had been previously treated with bisphosphonates (BPs), the drug used and time in treatment were recorded.

With regard to ONJ, the location of the lesions in the jaw was recorded and the presence of local and systemic risk factors that could contribute to the appearance of the lesions (dental extractions, implants, prosthesis or spontaneous appearance). In each case, orthopantomography was performed, and in three of the eight cases, a computerized tomography was performed. The presence of osteolysis, sclerosis and bone sequestration was recorded. The mean number of doses of Dmab was 3.4 \pm 2.2 .

Nine patients $(90 \%)$ had also been treated before with oral BPs, for an average of $44.7 \pm 25.11$ months. Four had been treated with ibandronate, three with risedronate and three with alendronate. In seven cases $(70 \%)$, the ONJ was located in the jaw. The most common local risk factor found was tooth extraction (six cases, 60\%); Only one case associated with dental implant was found. Three cases (30\%) had an intraoral and necrotic fistula and in 9 cases (90\%) bone exposure was observed. Regarding the radiographic changes observed, bone sclerosis was present in all cases. No sequestration was found in any case, osteolysis was present in six cases (60\%).

Stage 1 of ONJ was the most common, occurring in eight cases (80\%), and in all cases except one, conservative treatment was used with the healing of the lesions. One patient did not agree to be treated due to his age and concomitant systemic diseases. The follow-up was lost in two patients.

In the other study [6] the objective was to perform a systematic review that included the clinical cases published in the literature of people who develop ONM after DNM treatment. However, not all selected cases corresponded to people with osteoporosis. To do this, they conducted a review of the evidence using PubMed, Medline and Cochrane from January 2010 to May 2015. Articles should be case reports or series of clinical cases, in patients 18 years of age or older who used Dmab for the prevention of bone metastases and/ or treatment of osteoporosis and giant cell tumors or patients who had received some therapy for ONJ.

Based on the established criteria, 22 articles were selected. The parameters analyzed were: Type of

Table 3: Excluded studies and reasons for exclusion.

\begin{tabular}{|l|l|}
\hline Estudio (referencia bibliográfica) & Razones de exclusión \\
\hline Cooper [10] & Does not meet inclusion criteria \\
\hline Costa [11] & Does not meet inclusion criteria \\
\hline Dodson [12] & Does not meet inclusion criteria \\
\hline Henry [9] & Does not answer the question \\
\hline Khan [13] & Does not meet inclusion criteria \\
\hline Khan [5] & Does not meet inclusion criteria \\
\hline Khan [14] & Does not meet inclusion criteria \\
\hline Zavras [15] & Editorial \\
\hline Xue [16] & Does not meet inclusion criteria \\
\hline
\end{tabular}


disease treated, the characteristics of Dmab treatment, age and sex of the patient, site affected by ONJ, local and systemic risk factors and treatment.

Thirteen full case reports or case series articles were selected for this review. In total, 17 clinical cases were found. Of the 17 cases, only 8 were in patients who had received treatment with Dmab for osteoporosis or osteopenia (47.0\%) The majority of cases of ONJ occurred in the posterior mandibular area and only one of them in the posterior maxillary area. Previous treatment with BPs was the most frequent (in 6 of the 8 patients). One patient had received 3 different types of BPs (alendronate and risedronate for 6 years and zoledronate for 1 year). The second most frequent risk factor found was dental extractions ( 4 of the 8 cases). Of the 8 cases, in 4 of them the received treatment was not exposed. Of the remaining 4, only one of them required surgery and the rest remitted the lesions after conservative treatment.

\section{Discusion}

In the present study we have analyzed the risk of ONJ in patients that use Dmab for the treatment of osteoporosis. For the purpose of the present systematic literature review, we decided to include descriptive studies and case series and qualitative research studies.

We finally included a total of 2 studies. The quality of most of them was low.

ONJ has been observed in patients receiving denosumab at much higher doses than those prescribed for osteoporosis, typically as an antimetastatic agent for cancer [8]; however, it also has been reported in those receiving the normal doses for osteoporosis [5].

Some systemic factors such as previous treatment with BPs or dental extractions are associated with the development of osteonecrosis of the jaws in patients treated with denosumab (level of evidence 3) [6].

There are not enough studies that have evaluated the role of denosumab in the development of osteonecrosis of the jaws (level of evidence 3 ) $[6,7]$.

The incidence of ONJ in the FREEDOM trial extension was low $(0.18 \%)$, with most (six of eight) cases occurring after at least 4 years of exposure [9]. Dmab studies suggested that ONJ may be more likely to develop when Dmab is given to patients with risk factors such as dental extraction, poor oral hygiene, use of removable dental appliances, or chemotherapy [8].

The authors of the review found ONJ cases in seven trials in which Dmab was administered as an antimetastatic agent. These trials enrolled a total of 5072 patients who received $120 \mathrm{mg}$ of Dmab subcutaneously every 4 weeks or every 6 months, and ONJ was reported in 96 of them. Therefore, the incidence of ONJ was low, ranging from 0 to $2 \%$ over 13 to 21.4 months of treatment.

Several medical associations have published rec- ommendations to reduce the risk of this complication. In general, the guidelines include in their proposals a correct dental hygiene and revision by a dentist if poor dental health is objectified. If an invasive odontological procedure is planned -exodontia or implant- it is advisable to postpone the start of treatment until the surgical wound is completely healed.

There is some controversy about the attitude to be followed in patients who are already taking Dmab. Most guidelines advise non-oncological patients not to suspend the treatment. In a recent consensus document, in patients who are going to undergo extensive oral surgery and associated risk factors (diabetes, periodontal disease, immunodeficiencies, smoking), clinical judgment may advise temporarily suspending treatment. In this situation, unnecessary prolongation of the suspension should be avoided, especially in patients treated with Dmab. In case of a relevant adverse event such as osteonecrosis of the jaws, and although there is no scientific evidence to show that the withdrawal of the drug will improve the evolution of the process, the same document considers it prudent to suspend it and assess the indication of drugs with different mechanism of action [5].

In conclusion, not enough studies have been found that have evaluated the role of Dmab in the development of osteonecrosis (ONJ) of the jaws. Some systemic factors that have been associated with the development of ONJ are described in patients treated with Dmab, such as previous treatment with BPs or tooth extractions.

\section{References}

1. (2001) NIH Consensus Development Panel on osteoporosis prevention d, therapy M, 2000: Highlights of the conference. South Med J 94: 569-573.

2. Charopoulos I OS, Giannoudis PV (2011) The role and efficacy of denosumab in the treatment of osteoporosis: an update. Expert Opin Drug Saf 10: 205-217.

3. Sambrook P, Copper C (2006) Osteoporosis. Lancet 367: 2010-2018.

4. Cummings SR, San Martin J, McClung MR, Siris ES, Eastell $R$, et al. (2009) Denosumab for prevention of fractures in postmenopausal women with osteoporosis. N Engl J Med 361: 756-765.

5. Khan AA, Morrison A, Hanley DA, Felsenberg D, McCauley LK, et al. (2015) Diagnosis and management of osteonecrosis of the jaw: A systematic review and international consensus. J Bone Miner Res 30: 3-23.

6. de Oliveira CC, Brizeno LAC, De Sousa FB, Mota MRL, Alves APNN (2016) Osteonecrosis of the jaw induced by receptor activator of nuclear factor-kappa B ligand (Denosumab) - Review. Med Oral Patol Oral Cir Bucal 21: e431-e439.

7. Bagan J, Peydro A, Calvo J, Leopoldo $M$, Jiménez $Y$, et al. (2016) Medication-related osteonecrosis of the jaw associated with bisphosphonates and denosumab in osteoporosis. Oral Dis 22: 324-329. 
8. Boquete-Castro A, Gómez-Moreno G, Calvo-Guirado JL, Aguilar-Salvatierra A, Delgado-Ruiz RA (2015) Denosumab and osteonecrosis of the jaw. A systematic analysis of events reported in clinical trials. Clin Oral Implants Res 27: 367-375.

9. Bone HG, Wagman RB, Brandi ML, Brown JP, Chapurlat $R$, et al. (2017) 10 years of denosumab treatment in postmenopausal women with osteoporosis: results from the phase 3 randomised FREEDOM trial and open-label extension". Lancet Diabetes Endocrinol 5: 513-523.

10. Cooper C, Reginster JY, Cortet B, Diaz-Curiel M, Lorenc RS, et al. (2012) Long-term treatment of osteoporosis in postmenopausal women: A review from the European Society for Clinical and Economic Aspects of Osteoporosis and Osteoarthritis (ESCEO) and the International Osteoporosis Foundation (IOF). Curr Med Res Opin 28: 475-491.

11. Costa AG, Bilezikian JP (2015) How Long to Treat with Denosumab. Current Osteoporosis Reports 13: 415-420.
12. Dodson TB (2015) The Frequency of Medication-related Osteonecrosis of the Jaw and its Associated Risk Factors. Oral Maxillofac Surg Clin North Am 27: 509-516.

13. Khan A, Morrison A, Cheung A, Hashem W, Compston J (2016) Osteonecrosis of the jaw (ONJ): Diagnosis and management in 2015. Osteoporosis International 27: 853-859.

14. Khan AA, Morrison A, Kendler DL, Rizzoli R, Hanley DA, et al. (2017) Case-based review of osteonecrosis of the jaw $(\mathrm{ONJ})$ and application of the international recommendations for management from the international task force on ONJ. J Clin Densitom 20: 8-24.

15. Zavras Al (2012) Pharmacogenetics to screen for druginduced osteonecrosis of the jaw. Pharmacogenomics 13: 985-987.

16. Fei Xue, Rachel BW, Susan Yue, Shawna Smith, Tarun Arora, et al. (2015) Incidence rate of potential osteonecrosis of the jaw among women with postmenopausal osteoporosis treated with prolia or bisphosphonates. Arthritis Rheumatol 67. 